\title{
The method of upper and lower solutions for a class of fractional differential coupled systems
}

\author{
Huan Quan', Xiping Liu' ${ }^{1 *}$ and Mei Jia'
}

\section{"Correspondence:}

xipingliu@usst.edu.cn

${ }^{1}$ College of Science, University of

Shanghai for Science and

Technology, Shanghai 200093,

China

\begin{abstract}
In this paper, we investigate a class of nonlocal boundary value problems of nonlinear fractional functional differential coupled systems with state dependent delays. The method of upper and lower solutions is established and some new results for the multiplicity of solutions of the boundary value problem are obtained. An example is also presented to illustrate our main results.

Keywords: Differential functional differential coupled systems; State dependent delays; Boundary value problems; Riemann-Liouville fractional derivatives; Method of upper and lower solutions
\end{abstract}

\section{Introduction}

It is well know that fractional calculus is the generalization of classical calculus from integer to real numbers, even in the complex field. In the past decades, fractional differential equations have been widely used in various research fields, such as chemical engineering, automatic control, and thermoelasticity. In consequence, the theoretical researches of fractional differential equations have been highly valued by more and more scholars; see $[1-6]$.

When there are many state variables depending on each other in the system, the systems are often described as coupled systems; for details and examples, see [7-20] and the references therein. In recent years, the method of upper and lower solutions has played a more and more important role in the theoretical studies of differential equations. There are a large number of publications using the method of upper and lower solutions to study the existence and uniqueness of solutions of fractional differential equations; see [21-25] and the references therein.

In [10], the authors considered the three-point boundary value problems of nonlinear fractional coupled systems,

$$
\left\{\begin{array}{l}
D_{0^{+}}^{\alpha} u(t)=f\left(t, v(t), D_{0^{+}}^{\beta-1} v(t)\right), \quad 0<t<1, \\
D_{0^{+}}^{\beta} v(t)=g\left(t, u(t), D_{0^{+}}^{\alpha-1} u(t)\right), \quad 0<t<1, \\
u(0)=v(0)=0, \quad u(1)=\sigma_{1} u\left(\eta_{1}\right), \quad v(1)=\sigma_{2} v\left(\eta_{2}\right),
\end{array}\right.
$$

(c) The Author(s) 2021. This article is licensed under a Creative Commons Attribution 4.0 International License, which permits use, sharing, adaptation, distribution and reproduction in any medium or format, as long as you give appropriate credit to the original author(s) and the source, provide a link to the Creative Commons licence, and indicate if changes were made. The images or other third party material in this article are included in the article's Creative Commons licence, unless indicated otherwise in a credit line to the material. If material is not included in the article's Creative Commons licence and your intended use is not permitted by statutory regulation or exceeds the permitted use, you will need to obtain permission directly from the copyright holder. To view a copy of this licence, visit http://creativecommons.org/licenses/by/4.0/. 
where $D_{0^{+}}^{\alpha}$ is the Riemann-Liouville differentiation, $1<\alpha, \beta \leq 2,0<\eta_{1}, \eta_{2} \leq 1, \sigma_{1}, \sigma_{2}>$ $0, \sigma_{1} \eta_{1}^{\alpha-1}=\sigma_{2} \eta_{2}^{\beta-1}, f, g \in C\left([0,1] \times \mathbb{R}^{2}, \mathbb{R}\right)$. The existence theorems of two solutions of boundary value problems at resonance are given by using the coincidence degree theory.

In scientific research, if the system is assumed to be controlled by an equation containing the current state and the rate of change of state, then we consider ordinary or partial differential equations. However, in some applications, the system may also include the past state of the system. In this case, it would be more accurate to describe them by functional differential equations; see [26-28]. In recent years, the boundary value problems of the fractional functional differential equations have attracted the attention of researchers and many research results have been obtained; see [29-31].

At the same time, some problems may have already occurred for some time before we begin to study them, such as infectious diseases. In this way, we have to consider the values of state variables for the previous period of time. The purpose of this paper is to study the existence of multiple solutions for nonlocal boundary value problems of fractional functional differential coupled systems with time delays,

$$
\left\{\begin{array}{l}
D_{0^{+}}^{\alpha} u(t)+f\left(t, v(t), v_{t}\right)=0, \quad t \in(0,1), \\
D_{0^{+}}^{\beta} \nu(t)+g\left(t, u(t), u_{t}\right)=0, \quad t \in(0,1), \\
u(t)=\phi(t), \quad v(t)=\psi(t), \quad t \in[-\tau, 0], \\
D_{0^{+}}^{\gamma_{1}} u(1)=a D_{0^{+}}^{\gamma_{1}} u(\xi), \quad D_{0^{+}}^{\gamma_{2}} \nu(1)=b D_{0^{+}}^{\gamma_{2}} \nu(\eta),
\end{array}\right.
$$

where $0<\gamma_{1}, \gamma_{2} \leq 1,1+\gamma_{1} \leq \alpha \leq 2,1+\gamma_{2} \leq \beta \leq 2, \xi, \eta \in(0,1), a, b \in \mathbb{R} . D_{0^{+}}^{\alpha}, D_{0^{+}}^{\beta}, D_{0^{+}}^{\gamma_{1}}, D_{0^{+}}^{\gamma_{2}}$ are the Riemann-Liouville fractional derivative operators. The functions $f, g \in C([0,1] \times$ $\mathbb{R} \times C[-\tau, 0]) \cdot u_{t}=u(t+\theta), v_{t}=v(t+\theta), \theta \in[-\tau, 0], \phi, \psi \in C([-\tau, 0])$ and $\phi(0)=\psi(0)=0$. Some new results for the existence of at least three solutions for the coupled system are established by using upper and lower solutions methods.

\section{Preliminaries}

In this section, we present some necessary definitions and lemmas which will be used in the proof of our main results.

Definition 2.1 (see [1]) The Riemann-Liouville fractional integral of a function $h$ : $(0, \infty) \rightarrow \mathbb{R}$ of order $\alpha>0$ is given by

$$
I_{0^{+}}^{\alpha} h(t)=\frac{1}{\Gamma(\alpha)} \int_{0}^{t}(t-s)^{\alpha-1} h(s) d s,
$$

provided the right side is pointwise defined on $(0, \infty)$.

Definition 2.2 (see [1]) The Riemann-Liouville fractional derivative of order $\alpha>0$ of a continuous function $h:(0, \infty) \rightarrow \mathbb{R}$ is given by

$$
D_{0^{+}}^{\alpha} h(t)=D^{n} I_{0^{+}}^{n-\alpha} h(t)=\frac{1}{\Gamma(n-\alpha)}\left(\frac{d}{d t}\right)^{n} \int_{0}^{t} \frac{h(s)}{(t-s)^{\alpha-n+1}} d s,
$$

where $n=[\alpha]+1$ and $[\alpha]$ denote the integer parts of the real number $\alpha$, provided the right side is pointwise defined on $(0, \infty)$. 
Lemma 2.1 (see [1]) (1) If $h \in L(0,1), \rho>\sigma>0$, then

$D^{\sigma} I^{\rho} h(t)=I^{\rho-\sigma} h(t), D^{\sigma} I^{\sigma} h(t)=h(t)$.

(2) If $\rho>0, \lambda>0$, then

$$
D^{\rho} t^{\lambda-1}=\frac{\Gamma(\lambda)}{\Gamma(\lambda-\rho)} t^{\lambda-\rho-1} .
$$

Lemma 2.2 (see [1]) Assume $\alpha>0$, then the solution of the equation $D_{0^{+}}^{\alpha} h(t)=0$ is given by

$$
h(t)=c_{1} t^{\alpha-1}+c_{2} t^{\alpha-2}+\cdots+c_{n} t^{\alpha-n},
$$

where $c_{i} \in \mathbb{R}, i=1,2, \ldots, n$, and $n \in \mathbb{N}$ with $n-1<\alpha \leq n$.

For convenience, we denote

$$
\rho_{\alpha}:=\Gamma(\alpha)\left(1-a \xi^{\alpha-\gamma_{1}-1}\right), \quad \rho_{\beta}:=\Gamma(\beta)\left(1-b \eta^{\beta-\gamma_{2}-1}\right),
$$

and we always assume that $\rho_{\alpha}>0, \rho_{\beta}>0$.

Lemma 2.3 Suppose $a_{0}, b_{0}$ are constants. Then for any given functions $x, y \in C[0,1]$, the boundary value problem of the linear fractional differential system

$$
\left\{\begin{array}{l}
D_{0^{+}}^{\alpha} u(t)+x(t)=0, \quad t \in(0,1), \\
D_{0^{+}}^{\beta} \nu(t)+y(t)=0, \quad t \in(0,1), \\
u(0)=v(0)=0, \\
D_{0^{+}}^{\gamma_{1}} u(1)=a D_{0^{+}}^{\gamma_{1}} u(\xi)-a_{0}, \quad D_{0^{+}}^{\gamma_{2}} \nu(1)=b D_{0^{+}}^{\gamma_{2}} \nu(\eta)-b_{0}
\end{array}\right.
$$

has a unique solution $(u, v)=(u(t), v(t))$ as

$$
\begin{aligned}
& u(t)=\int_{0}^{1} G_{\alpha}(t, s) x(s) d s-\frac{1}{\rho_{\alpha}} a_{0} \Gamma\left(\alpha-\gamma_{1}\right) t^{\alpha-1}, \\
& v(t)=\int_{0}^{1} G_{\beta}(t, s) y(s) d s-\frac{1}{\rho_{\beta}} b_{0} \Gamma\left(\beta-\gamma_{2}\right) t^{\beta-1},
\end{aligned}
$$

where

$$
G_{\alpha}(t, s)=\frac{1}{\rho_{\alpha}}\left\{\begin{array}{c}
t^{\alpha-1}(1-s)^{\alpha-\gamma_{1}-1}-\left(1-a \xi^{\alpha-\gamma_{1}-1}\right)(t-s)^{\alpha-1}-a t^{\alpha-1}(\xi-s)^{\alpha-\gamma_{1}-1}, \\
0 \leq s \leq \min \{t, \xi\} \leq 1, \\
t^{\alpha-1}(1-s)^{\alpha-\gamma_{1}-1}-a t^{\alpha-1}(\xi-s)^{\alpha-\gamma_{1}-1} \\
0 \leq t<s \leq \xi \leq 1, \\
t^{\alpha-1}(1-s)^{\alpha-\gamma_{1}-1}-\left(1-a \xi^{\alpha-\gamma_{1}-1}\right)(t-s)^{\alpha-1}, \\
0 \leq \xi \leq s<t \leq 1, \\
t^{\alpha-1}(1-s)^{\alpha-\gamma_{1}-1} \\
0 \leq \max \{t, \xi\} \leq s \leq 1,
\end{array}\right.
$$




$$
G_{\beta}(t, s)=\frac{1}{\rho_{\beta}}\left\{\begin{array}{l}
t^{\beta-1}(1-s)^{\beta-\gamma_{2}-1}-\left(1-b \eta^{\beta-\gamma_{2}-1}\right)(t-s)^{\beta-1}-b t^{\beta-1}(\eta-s)^{\beta-\gamma_{2}-1} \\
0 \leq s \leq \min \{t, \eta\} \leq 1 \\
t^{\beta-1}(1-s)^{\beta-\gamma_{2}-1}-b t^{\beta-1}(\eta-s)^{\beta-\gamma_{2}-1} \\
0 \leq t<s \leq \eta \leq 1 \\
t^{\beta-1}(1-s)^{\beta-\gamma_{2}-1}-\left(1-b \eta^{\beta-\gamma_{2}-1}\right)(t-s)^{\beta-1} \\
0 \leq \eta \leq s<t \leq 1 \\
t^{\beta-1}(1-s)^{\beta-\gamma_{2}-1} \\
0 \leq \max \{t, \eta\} \leq s \leq 1
\end{array}\right.
$$

Proof Assume $(u, v)=(u(t), v(t))$ is a solution of the linear fractional system (2.2). By applying Lemma 2.2,

$$
\begin{aligned}
& u(t)=-I_{0^{+}}^{\alpha} x(t)+c_{1} t^{\alpha-1}+c_{2} t^{\alpha-2}, \\
& v(t)=-I_{0^{+}}^{\beta} y(t)+d_{1} t^{\beta-1}+d_{2} t^{\beta-2},
\end{aligned}
$$

where $c_{1}, c_{2}, d_{1}, d_{2} \in \mathbb{R}$. In view of $u(0)=v(0)=0$, we have $c_{2}=0, d_{2}=0$. Then

$$
\begin{aligned}
& u(t)=-I_{0^{+}}^{\alpha} x(t)+c_{1} t^{\alpha-1}, \\
& v(t)=-I_{0^{+}}^{\beta} y(t)+d_{1} t^{\beta-1} .
\end{aligned}
$$

By Lemma 2.1, we have

$$
\begin{aligned}
& D_{0^{+}}^{\gamma_{1}} u(t)=-D_{0^{+}}^{\gamma_{1}} I_{0^{+}}^{\alpha} x(t)+c_{1} D_{0^{+}}^{\gamma_{1}} t^{\alpha-1}=-I_{0^{+}}^{\alpha-\gamma_{1}} x(t)+c_{1} \frac{\Gamma(\alpha)}{\Gamma\left(\alpha-\gamma_{1}\right)} t^{\alpha-\gamma_{1}-1}, \\
& D_{0^{+}}^{\gamma_{2}} \nu(t)=-D_{0^{+}}^{\gamma_{2}} I_{0^{+}}^{\beta} y(t)+d_{1} D_{0^{+}}^{\gamma_{2}} t^{\beta-1}=-I_{0^{+}}^{\beta-\gamma_{2}} y(t)+d_{1} \frac{\Gamma(\beta)}{\Gamma\left(\beta-\gamma_{2}\right)} t^{\beta-\gamma_{2}-1} .
\end{aligned}
$$

And

$$
\begin{aligned}
& D_{0^{+}}^{\gamma_{1}} u(1)=-\frac{1}{\Gamma\left(\alpha-\gamma_{1}\right)} \int_{0}^{1}(1-s)^{\alpha-\gamma_{1}-1} x(s) d s+c_{1} \frac{\Gamma(\alpha)}{\Gamma\left(\alpha-\gamma_{1}\right)}, \\
& D_{0^{+}}^{\gamma_{1}} u(\xi)=-\frac{1}{\Gamma\left(\alpha-\gamma_{1}\right)} \int_{0}^{\xi}(\xi-s)^{\alpha-\gamma_{1}-1} x(s) d s+c_{1} \frac{\Gamma(\alpha)}{\Gamma\left(\alpha-\gamma_{1}\right)} \xi^{\alpha-\gamma_{1}-1}, \\
& D_{0^{+}}^{\gamma_{2}} \nu(1)=-\frac{1}{\Gamma\left(\beta-\gamma_{2}\right)} \int_{0}^{1}(1-s)^{\beta-\gamma_{2}-1} y(s) d s+d_{1} \frac{\Gamma(\beta)}{\Gamma\left(\beta-\gamma_{2}\right)}, \\
& D_{0^{+}}^{\gamma_{2}} \nu(\eta)=-\frac{1}{\Gamma\left(\beta-\gamma_{2}\right)} \int_{0}^{\eta}(\eta-s)^{\beta-\gamma_{2}-1} y(s) d s+d_{1} \frac{\Gamma(\beta)}{\Gamma\left(\beta-\gamma_{2}\right)} \eta^{\beta-\gamma_{2}-1} .
\end{aligned}
$$

By the boundary conditions $D_{0^{+}}^{\gamma_{1}} u(1)=a D_{0^{+}}^{\gamma_{1}} u(\xi)-a_{0}, D_{0^{+}}^{\gamma_{2}} v(1)=b D_{0^{+}}^{\gamma_{2}} \nu(\eta)-b_{0}$, we have

$$
\begin{aligned}
& c_{1}=\frac{1}{\rho_{\alpha}}\left(\int_{0}^{1}(1-s)^{\alpha-\gamma_{1}-1} x(s) d s-a \int_{0}^{\xi}(\xi-s)^{\alpha-\gamma_{1}-1} x(s) d s-a_{0} \Gamma\left(\alpha-\gamma_{1}\right)\right), \\
& d_{1}=\frac{1}{\rho_{\beta}}\left(\int_{0}^{1}(1-s)^{\beta-\gamma_{2}-1} y(s) d s-b \int_{0}^{\eta}(\eta-s)^{\beta-\gamma_{2}-1} y(s) d s-b_{0} \Gamma\left(\beta-\gamma_{2}\right)\right) .
\end{aligned}
$$


So,

$$
\begin{aligned}
u(t)= & -\frac{1}{\Gamma(\alpha)} \int_{0}^{t}(t-s)^{\alpha-1} x(s) d s+\frac{t^{\alpha-1}}{\rho_{\alpha}}\left(\int_{0}^{1}(1-s)^{\alpha-\gamma_{1}-1} x(s) d s\right. \\
& \left.-a \int_{0}^{\xi}(\xi-s)^{\alpha-\gamma_{1}-1} x(s) d s-a_{0} \Gamma\left(\alpha-\gamma_{1}\right)\right) \\
= & \int_{0}^{1} G_{\alpha}(t, s) x(s) d s-\frac{1}{\rho_{\alpha}} a_{0} \Gamma\left(\alpha-\gamma_{1}\right) t^{\alpha-1} .
\end{aligned}
$$

Similarly,

$$
v(t)=\int_{0}^{1} G_{\beta}(t, s) y(s) d s-\frac{1}{\rho_{\beta}} b_{0} \Gamma\left(\beta-\gamma_{2}\right) t^{\beta-1} .
$$

On the other hand, we can easily see that $(u, v)=(u(t), v(t))$ is a solution of the linear fractional system (2.2) if $u=u(t), v=v(t)$ for $t \in[0,1]$ satisfy (2.3) and (2.4), respectively.

Lemma 2.4 Assume $\rho_{\alpha}>0, \rho_{\beta}>0$, then $G_{\alpha}(t, s), G_{\beta}(t, s)$ defined by (2.5) and (2.6), respectively, have the following properties:

(1) $G_{\alpha}(t, s), G_{\beta}(t, s)$ are continuous on $(t, s) \in[0,1] \times[0,1]$;

(2) $0<G_{\alpha}(t, s) \leq \max _{0 \leq t \leq 1} G_{\alpha}(t, s) \leq w_{1}(s), t, s \in(0,1)$;

(3) $0<G_{\beta}(t, s) \leq \max _{0 \leq t \leq 1} G_{\beta}(t, s) \leq w_{2}(s), t, s \in(0,1)$, where $w_{1}(s)=\frac{(1-s)^{\alpha-\gamma_{1}-1}}{\Gamma(\alpha)}\left(s^{\alpha-1}+\right.$ $\left.\frac{a \xi^{\alpha-\gamma_{1}-1}}{1-a \xi^{\alpha-\gamma_{1}-1}}\right), w_{2}(s)=\frac{(1-s)^{\beta-\gamma_{2}-1}}{\Gamma(\beta)}\left(s^{\beta-1}+\frac{b \eta^{\beta-\gamma_{2}-1}}{1-b \eta^{\beta-\gamma_{2}-1}}\right)$.

Proof (1) The continuity of $G_{\alpha}(t, s), G_{\beta}(t, s)$ is obvious by (2.5) and (2.6).

(2) Let

$$
g_{1}(t, s)=\frac{1}{\Gamma(\alpha)}\left(t^{\alpha-1}(1-s)^{\alpha-\gamma_{1}-1}-(t-s)^{\alpha-1}\right), \quad t, s \in(0,1) .
$$

It is clear that

$$
\begin{aligned}
g_{1}(t, s) & =\frac{1}{\Gamma(\alpha)}\left(t^{\alpha-1}(1-s)^{\alpha-\gamma_{1}-1}-(t-s)^{\alpha-1}\right) \\
& >\frac{1}{\Gamma(\alpha)}\left(t^{\alpha-1}(1-s)^{\alpha-1}-(t-s)^{\alpha-1}\right)>0, \quad t, s \in(0,1),
\end{aligned}
$$

and for $0<s \leq t<1$,

$$
\begin{aligned}
\frac{\partial g_{1}(t, s)}{\partial t} & =\frac{\alpha-1}{\Gamma(\alpha)}\left(t^{\alpha-2}(1-s)^{\alpha-\gamma_{1}-1}-(t-s)^{\alpha-2}\right) \\
& =\frac{1}{\Gamma(\alpha-1)} t^{\alpha-2}\left((1-s)^{\alpha-\gamma_{1}-1}-\left(1-\frac{s}{t}\right)^{\alpha-2}\right)<0,
\end{aligned}
$$

and for $0<t \leq s<1$,

$$
\begin{aligned}
\frac{\partial g_{1}(t, s)}{\partial t} & =\frac{\alpha-1}{\Gamma(\alpha)}\left(t^{\alpha-2}(1-s)^{\alpha-\gamma_{1}-1}-(t-s)^{\alpha-2}\right) \\
& =\frac{1}{\Gamma(\alpha-1)} t^{\alpha-2}\left((1-s)^{\alpha-\gamma_{1}-1}-\left(1-\frac{s}{t}\right)^{\alpha-2}\right)>0,
\end{aligned}
$$


which imply that $g_{1}(t, s)>0$, and $g_{1}(t, s)$ is decreasing with respect to $t$ as $s \leq t$ and increasing with respect to $t$ as $t \leq s$.

So,

$$
\begin{aligned}
& \max _{0 \leq t \leq 1} g_{1}(t, s)=g_{1}(s, s)=\frac{1}{\Gamma(\alpha)} s^{\alpha-1}(1-s)^{\alpha-\gamma_{1}-1}, \\
& \min _{0 \leq t \leq 1} g_{1}(t, s)=g_{1}(1, s) \geq 0 .
\end{aligned}
$$

For $0<s \leq \min \{t, \xi\}<1$,

$$
\begin{aligned}
G_{\alpha}(t, s)= & \frac{1}{\rho_{\alpha}}\left(t^{\alpha-1}(1-s)^{\alpha-\gamma_{1}-1}-\left(1-a \xi^{\alpha-\gamma_{1}-1}\right)(t-s)^{\alpha-1}-a t^{\alpha-1}(\xi-s)^{\alpha-\gamma_{1}-1}\right) \\
= & \frac{1}{\Gamma(\alpha)}\left(1+\frac{a \xi^{\alpha-\gamma_{1}-1}}{1-a \xi^{\alpha-\gamma_{1}-1}}\right) t^{\alpha-1}(1-s)^{\alpha-\gamma_{1}-1} \\
& -\frac{1}{\Gamma(\alpha)}(t-s)^{\alpha-1}-\frac{1}{\rho_{\alpha}} a t^{\alpha-1}(\xi-s)^{\alpha-\gamma_{1}-1} \\
= & \frac{1}{\Gamma(\alpha)}\left(t^{\alpha-1}(1-s)^{\alpha-\gamma_{1}-1}-(t-s)^{\alpha-1}\right) \\
& +\frac{a t^{\alpha-1}}{\rho_{\alpha}}\left(\xi^{\alpha-\gamma_{1}-1}(1-s)^{\alpha-\gamma_{1}-1}-(\xi-s)^{\alpha-\gamma_{1}-1}\right) \\
= & g_{1}(t, s)+\frac{a t^{\alpha-1}}{\rho_{\alpha}}\left(\xi^{\alpha-\gamma_{1}-1}(1-s)^{\alpha-\gamma_{1}-1}-(\xi-s)^{\alpha-\gamma_{1}-1}\right) \\
\geq & g_{1}(t, s)+\frac{a t^{\alpha-1}}{\left(1-a \xi^{\alpha-\gamma_{1}-1}\right)} g_{1}(\xi, s)>0 .
\end{aligned}
$$

In a similar way we show $G_{\alpha}(t, s)>0$ for $0<t<s \leq \xi<1$ or $0<\xi \leq s<t<1$ or $0<$ $\max \{t, \xi\} \leq s<1$. Hence, $G_{\alpha}(t, s)>0$ for $t, s \in(0,1)$, and it is obvious $G_{\alpha}(1, s)>0$ for $s \in$ $(0,1)$.

Next, we will prove that $\max _{0 \leq t \leq 1} G_{\alpha}(t, s) \leq w_{1}(s)$.

If $0 \leq s \leq \min \{t, \xi\} \leq 1$, we have

$$
\begin{aligned}
\max _{s \leq t \leq 1} G_{\alpha}(t, s) & =\max _{s \leq t \leq 1}\left(g_{1}(t, s)+\frac{1}{\rho_{\alpha}}\left(a t^{\alpha-1}\left(\xi^{\alpha-\gamma_{1}-1}(1-s)^{\alpha-\gamma_{1}-1}-(\xi-s)^{\alpha-\gamma_{1}-1}\right)\right)\right) \\
& \leq g_{1}(s, s)+\frac{1}{\rho_{\alpha}} a \xi^{\alpha-\gamma_{1}-1}(1-s)^{\alpha-\gamma_{1}-1}=w_{1}(s) .
\end{aligned}
$$

If $0<\xi \leq s<t \leq 1$, by (2.7), we get

$$
\begin{aligned}
& \max _{s \leq t \leq 1} G_{\alpha}(t, s) \\
& =\max _{s \leq t \leq 1} \frac{1}{\rho_{\alpha}}\left(t^{\alpha-1}(1-s)^{\alpha-\gamma_{1}-1}-\left(1-a \xi^{\alpha-\gamma_{1}-1}\right)(t-s)^{\alpha-1}\right) \\
& =\max _{s \leq t \leq 1}\left(\frac{1}{\Gamma(\alpha)}\left(1+\frac{a \xi^{\alpha-\gamma_{1}-1}}{1-a \xi^{\alpha-\gamma_{1}-1}}\right) t^{\alpha-1}(1-s)^{\alpha-\gamma_{1}-1}-\frac{1}{\Gamma(\alpha)}(t-s)^{\alpha-1}\right) \\
& =\max _{s \leq t \leq 1}\left(\frac{1}{\Gamma(\alpha)}\left(t^{\alpha-1}(1-s)^{\alpha-\gamma_{1}-1}-(t-s)^{\alpha-1}\right)+\frac{1}{\rho_{\alpha}} a \xi^{\alpha-\gamma_{1}-1} t^{\alpha-1}(1-s)^{\alpha-\gamma_{1}-1}\right)
\end{aligned}
$$




$$
\begin{aligned}
& =\max _{s \leq t \leq 1}\left(g_{1}(t, s)+\frac{1}{\rho_{\alpha}} a \xi^{\alpha-\gamma_{1}-1} t^{\alpha-1}(1-s)^{\alpha-\gamma_{1}-1}\right) \\
& \leq \frac{1}{\Gamma(\alpha)} s^{\alpha-1}(1-s)^{\alpha-\gamma_{1}-1}+\frac{1}{\rho_{\alpha}} a \xi^{\alpha-\gamma_{1}-1}(1-s)^{\alpha-\gamma_{1}-1} \\
& \leq w_{1}(s) .
\end{aligned}
$$

If $0 \leq t<s \leq \xi<1$, we can see that

$$
\begin{aligned}
\max _{0 \leq t \leq s} G_{\alpha}(t, s) & =\max _{0 \leq t \leq 1} \frac{1}{\rho_{\alpha}}\left(t^{\alpha-1}(1-s)^{\alpha-\gamma_{1}-1}-a t^{\alpha-1}(\xi-s)^{\alpha-\gamma_{1}-1}\right) \\
& =\max _{0 \leq t \leq s}\left(\frac{t^{\alpha-1}(1-s)^{\alpha-\gamma_{1}-1}}{\Gamma(\alpha)}+\frac{1}{\rho_{\alpha}} a t^{\alpha-1}\left(\xi^{\alpha-\gamma_{1}-1}(1-s)^{\alpha-\gamma_{1}-1}-(\xi-s)^{\alpha-1}\right)\right) \\
& \leq w_{1}(s) .
\end{aligned}
$$

If $0 \leq \max \{t, \xi\} \leq s \leq 1$, then

$$
\begin{aligned}
\max _{0 \leq t \leq s} G_{\alpha}(t, s) & =\max _{0 \leq t \leq s} \frac{1}{\rho_{\alpha}} t^{\alpha-1}(1-s)^{\alpha-\gamma_{1}-1} \\
& =\max _{0 \leq t \leq s} \frac{1}{\Gamma(\alpha)}\left(1+\frac{a \xi^{\alpha-\gamma_{1}-1}}{1-a \xi^{\alpha-\gamma_{1}-1}}\right) t^{\alpha-1}(1-s)^{\alpha-\gamma_{1}-1} \\
& \leq w_{1}(s) .
\end{aligned}
$$

Hence,

$$
0<G_{\alpha}(t, s) \leq \max _{0 \leq t \leq 1} G_{\alpha}(t, s) \leq w_{1}(s), \quad s, t \in(0,1) .
$$

(3) Similarly, we can prove the inequality.

By Lemma 2.3, let $a_{0}=b_{0}=0$, and we can get the following lemma.

Lemma 2.5 The fractional differential coupled system (1.1) is equivalent to the systems of integral systems

$$
\begin{aligned}
& u(t)= \begin{cases}\int_{0}^{1} G_{\alpha}(t, s) f\left(s, v(s), v_{s}\right) d s, & t \in(0,1], \\
\phi(t), & t \in[-\tau, 0],\end{cases} \\
& v(t)= \begin{cases}\int_{0}^{1} G_{\beta}(t, s) g\left(s, u(s), u_{s}\right) d s, & t \in(0,1], \\
\psi(t), & t \in[-\tau, 0] .\end{cases}
\end{aligned}
$$

Let $E=\{(u, v): u, v \in C[-\tau, 1]\}$ and be endowed with norm

$$
\|(u, v)\|_{E}=\max \left\{\max _{t \in[-\tau, 1]}|u(t)|, \max _{t \in[-\tau, 1]}|v(t)|\right\}
$$

and $C[-\tau, 0]$ endowed with the norm $\|x\|_{\tau}=\max _{t \in[-\tau, 0]}|x(t)|$. Then $\left(E,\|\cdot\|_{E}\right)$ and $\left(C[-\tau, 0],\|\cdot\|_{\tau}\right)$ are Banach spaces. 
Let

$$
E_{0}=\{(r, z) \in E:(r(t), z(t)) \equiv(0,0), t \in[-\tau, 0]\}
$$

be endowed with norm

$$
\|(r, z)\|_{E_{0}}=\max \left\{\max _{t \in[-\tau, 1]}|r(t)|, \max _{t \in[-\tau, 1]}|z(t)|\right\}=\max \left\{\max _{t \in[0,1]}|r(t)|, \max _{t \in[0,1]}|z(t)|\right\}
$$

and $P=\left\{(r, z) \in E_{0}: r(t) \geq 0, z(t) \geq 0, t \in(0,1]\right\}$. Obviously, $E_{0} \subset E$ and $\left(E_{0},\|\cdot\|_{E_{0}}\right)$ is a Banach space, and $P \subset E_{0}$ is a normal solid cone.

For $\left(u_{1}, v_{1}\right),\left(u_{2}, v_{2}\right) \in E_{0},\left(u_{1}, v_{1}\right) \preceq\left(u_{2}, v_{2}\right)$ if and only if $\left(u_{2}-u_{1}, v_{2}-v_{1}\right) \in P$. Hence, $\left(E_{0}, \preceq\right)$ is a partial order Banach space. We denote $\left(u_{1}, v_{1}\right) \prec\left(u_{2}, v_{2}\right)$ if $\left(u_{1}, v_{1}\right) \preceq\left(u_{2}, v_{2}\right) \in E_{0}$ and $\left(u_{1}, v_{1}\right) \neq\left(u_{2}, v_{2}\right)$; we denote $\left(u_{1}, v_{1}\right) \prec \prec\left(u_{2}, v_{2}\right)$ if $\left(u_{2}-u_{1}, v_{2}-v_{1}\right) \in P^{\circ}$.

Redefine functions $\phi(t)$ and $\psi(t)$ on $t \in[0,1]$. Let $\phi(t)=\psi(t)=0, t \in[0,1]$. Obviously, $\phi, \psi \in E$.

For any $(r, z) \in E_{0}$, let

$$
u(t)=\phi(t)+r(t)= \begin{cases}r(t), & t \in[0,1], \\ \phi(t), & t \in[-\tau, 0],\end{cases}
$$

and

$$
v(t)=\psi(t)+z(t)= \begin{cases}z(t), & t \in[0,1], \\ \psi(t), & t \in[-\tau, 0] .\end{cases}
$$

Then for $t \in[0,1], u_{t}=\phi_{t}+r_{t}=\phi(t+\theta)+r(t+\theta), v_{t}=\psi_{t}+z_{t}=\psi(t+\theta)+z(t+\theta), \theta \in[-\tau, 0]$. It is easy to see that the following lemma holds.

Lemma $2.6(u, v) \in E$ is a solution of systems (2.9) and (2.10) if and only if $(r, z) \in E_{0}$ is a solution of the following integral systems:

$$
r(t)= \begin{cases}0, & t \in[-\tau, 0], \\ \int_{0}^{1} G_{\alpha}(t, s) f\left(s, z(s), \psi_{s}+z_{s}\right) d s, & t \in(0,1],\end{cases}
$$

and

$$
z(t)= \begin{cases}0, & t \in[-\tau, 0], \\ \int_{0}^{1} G_{\beta}(t, s) g\left(s, r(s), \phi_{s}+r_{s}\right) d s, & t \in(0,1],\end{cases}
$$

which implies that $(u, v) \in E$ is a solution of system (1.1) if and only if $(r, z) \in E_{0}$ is a solution of the following coupled system:

$$
\left\{\begin{array}{l}
D_{0^{+}}^{\alpha} r(t)+f\left(t, z(t), \psi_{t}+z_{t}\right)=0, \quad t \in(0,1), \\
D_{0^{+}}^{\beta} z(t)+g\left(t, r(t), \phi_{t}+r_{t}\right)=0, \quad t \in(0,1), \\
D_{0^{+}}^{\gamma_{1}} r(1)=a D_{0^{+}}^{\gamma_{1}} r(\xi), \quad D_{0^{+}}^{\gamma_{2}} z(1)=b D_{0^{+}}^{\gamma_{2}} z(\eta), \\
r(t)=0, \quad z(t)=0, \quad t \in[-\tau, 0] .
\end{array}\right.
$$


Define an operator $T: E_{0} \rightarrow E_{0}$ by

$$
T(r, z)=(A(r, z), B(r, z)),
$$

where

$$
\begin{aligned}
& A(r, z)(t)= \begin{cases}0, & t \in[-\tau, 0], \\
\int_{0}^{1} G_{\alpha}(t, s) f\left(s, z(s), \psi_{s}+z_{s}\right) d s, & t \in(0,1],\end{cases} \\
& B(r, z)(t)= \begin{cases}0, & t \in[-\tau, 0], \\
\int_{0}^{1} G_{\beta}(t, s) g\left(s, r(s), \phi_{s}+r_{s}\right) d s, & t \in(0,1] .\end{cases}
\end{aligned}
$$

It is clear that the Lemma 2.7 holds.

Lemma 2.7 A solution of the system (1.1) on $E$ is equivalent to a fixed point of operator $T$ on $E_{0}$.

Lemma 2.8 The operator $T: E_{0} \rightarrow E_{0}$ is completely continuous.

Proof Firstly, we prove that operator $T$ is continuous on $E_{0}$.

Let $\left\{r_{n}, z_{n}\right\} \subset E_{0},(r, z) \in E_{0}$ such that $\left\|\left(r_{n}, z_{n}\right)-(r, z)\right\|_{E_{0}} \rightarrow 0$ as $n \rightarrow \infty$. Then, there exists a constant $M_{0} \geq 0$ such that $\left\|\left(r_{n}, z_{n}\right)\right\|_{E_{0}} \leq M_{0}$ for $n=1,2, \ldots$ and $\|(r, z)\|_{E_{0}} \leq M_{0}$, and then $\left\|r_{n t}\right\|_{\tau} \leq M_{0},\left\|z_{n t}\right\|_{\tau} \leq M_{0},\left\|r_{t}\right\|_{\tau} \leq M_{0}$ and $\left\|z_{t}\right\|_{\tau} \leq M_{0}$ for $t \in[0,1]$. Due to $\theta \in[-\tau, 0]$, we have $\theta+t \in[-\tau, 1]$ for $t \in[0,1]$. Therefore, we have $\left\|\left(r_{n t}, z_{n t}\right)-\left(r_{t}, z_{t}\right)\right\|_{E_{0}} \rightarrow 0$ as $n \rightarrow \infty$.

By the continuity of $f, g$,

$$
\begin{aligned}
& \lim _{n \rightarrow \infty}\left|f\left(t, z_{n}(t), \psi_{t}+z_{n t}\right)-f\left(t, z(t), \psi_{t}+z_{t}\right)\right|=0 \quad \text { and } \\
& \lim _{n \rightarrow \infty}\left|g\left(t, r_{n}(t), \phi_{t}+r_{n t}\right)-g\left(t, r(t), \phi_{t}+r_{t}\right)\right|=0 .
\end{aligned}
$$

And there exist constants $M_{1}, M_{2}>0$ such that

$$
\left|f\left(t, z_{n}(t), \psi_{t}+z_{n t}\right)\right| \leq M_{1} \quad \text { and } \quad\left|g\left(t, r_{n}(t), \phi_{t}+r_{n t}\right)\right| \leq M_{2}, \quad t \in[0,1], n=1,2, \ldots
$$

and

$$
\left|f\left(t, z(t), \psi_{t}+z_{t}\right)\right| \leq M_{1}, \quad\left|g\left(t, r(t), \phi_{t}+r_{t}\right)\right| \leq M_{2}
$$

Then

$$
\begin{aligned}
& \left|f\left(t, z_{n}(t), \psi_{t}+z_{n t}\right)-f\left(t, z(t), \psi_{t}+z_{t}\right)\right| \leq 2 M_{1} \quad \text { and } \\
& \left|g\left(t, r_{n}(t), \phi_{t}+r_{n t}\right)-g\left(t, r(t), \phi_{t}+r_{t}\right)\right| \leq 2 M_{2} .
\end{aligned}
$$

It follows from Lemma 2.4 that for $t \in[-\tau, 1]$

$$
\begin{aligned}
\left|A\left(r_{n}, z_{n}\right)(t)-A(r, z)(t)\right| & \leq\left|\int_{0}^{1} G_{\alpha}(t, s)\left(f\left(t, z_{n}(s), \psi_{s}+z_{n s}\right)-f\left(s, z(s), \psi_{s}+z_{s}\right)\right)\right| d s \\
& \leq \int_{0}^{1} w_{1}(s)\left|f\left(t, z_{n}(s), \psi_{s}+z_{n s}\right)-f\left(s, z(s), \psi_{s}+z_{s}\right)\right| d s \rightarrow 0
\end{aligned}
$$


and

$$
\begin{aligned}
\left|B\left(r_{n}, z_{n}\right)(t)-B(r, z)(t)\right| & \leq\left|\int_{0}^{1} G_{\beta}(t, s)\left(g\left(t, r_{n}(s), \phi_{s}+r_{n s}\right)-f\left(s, r(s), \phi_{s}+r_{s}\right)\right)\right| d s \\
& \leq \int_{0}^{1} w_{2}(s)\left|g\left(t, r_{n}(s), \phi_{s}+r_{n s}\right)-g\left(s, r(s), \phi_{s}+r_{s}\right)\right| d s \rightarrow 0 .
\end{aligned}
$$

By Lebesgue's dominated convergence theorem, as $n \rightarrow \infty$,

$$
A\left(r_{n}, z_{n}\right) \rightarrow A(r, z), \quad B\left(r_{n}, z_{n}\right) \rightarrow B(r, z) .
$$

Consequently, the operator $T$ is continuous.

Assume that $S \subset E_{0}$ is a bounded set, and there exists a constant $l_{1}>0$ such that we have $\|(r, z)\|_{E_{0}} \leq l_{1}$ for any $(r, z) \in S$. There exist constants $M_{3}>0$ and $M_{4}>0$ such that

$$
\left|f\left(t, z(t), \psi_{t}+z_{t}\right)\right| \leq M_{3}, \quad\left|g\left(t, r(t), \phi_{t}+r_{t}\right)\right| \leq M_{4}
$$

Therefore, by Lemma 2.4, we have

$$
\begin{aligned}
|A(r, z)(t)| & \leq \int_{0}^{1}\left|G_{\alpha}(t, s)\right|\left|f\left(s, z(s), \psi_{s}+z_{s}\right)\right| d s \leq M_{3} \int_{0}^{1} \omega_{1}(s) d s \\
& \leq \frac{M_{3}}{\Gamma(\alpha)} \int_{0}^{1}\left(s^{\alpha-1}+\frac{a \xi^{\alpha-\gamma_{1}-1}(1-s)^{\alpha-\gamma_{1}-1}}{1-a \xi^{\alpha-\gamma_{1}-1}}\right) d s \\
& =\left(\frac{1}{\Gamma(\alpha+1)}+\frac{a \xi^{\alpha-\gamma_{1}-1}}{\left(\alpha-\gamma_{1}\right) \rho_{\alpha}}\right) M_{3} .
\end{aligned}
$$

Similarly, we can prove that

$$
|B(r, z)(t)| \leq\left(\frac{1}{\Gamma(\beta+1)}+\frac{b \eta^{\beta-\gamma_{2}-1}}{\left(\beta-\gamma_{2}\right) \rho_{\beta}}\right) M_{4}
$$

Therefore, there exists a constant $l>0$, such that $\|T(r, z)\|_{E_{0}} \leq l$ for $(r, z) \in S$. So $T(S)$ is uniformly bounded.

By the continuity of $G_{\alpha}(t, s)$ and $G_{\beta}(t, s)$ on $[0,1] \times[0,1]$, we have $G_{\alpha}(t, s)$ and $G_{\beta}(t, s)$ are uniformly continuous on $[0,1] \times[0,1]$. Therefore, for any $\varepsilon>0$, there exists $\delta>0$ such that

$$
\left|G_{\alpha}\left(t_{1}, s\right)-G_{\alpha}\left(t_{2}, s\right)\right|<\frac{\varepsilon}{M_{3}}, \quad\left|G_{\beta}\left(t_{1}, s\right)-G_{\beta}\left(t_{2}, s\right)\right|<\frac{\varepsilon}{M_{4}}
$$

whenever $t_{1}, t_{2}, s \in[0,1]$ and $\left|t_{1}-t_{2}\right|<\delta$.

If $t_{1}, t_{2} \in[0,1]$ and $\left|t_{1}-t_{2}\right|<\delta$, we get

$$
\begin{aligned}
\left|A(r, z)\left(t_{1}\right)-A(r, z)\left(t_{2}\right)\right| & =\left|\int_{0}^{1}\left(G_{\alpha}\left(t_{1}, s\right)-G_{\alpha}\left(t_{2}, s\right)\right) f\left(s, z(s), \phi_{s}+z_{s}\right) d s\right| \\
& \leq M_{3} \int_{0}^{1}\left|G_{\alpha}\left(t_{1}, s\right)-G_{\alpha}\left(t_{2}, s\right)\right| d s<\varepsilon .
\end{aligned}
$$


Similarly,

$$
\left|B(r, z)\left(t_{1}\right)-B(r, z)\left(t_{2}\right)\right|<\varepsilon
$$

And if $t_{1}, t_{2} \in[-\tau, 0]$ and $\left|t_{1}-t_{2}\right|<\delta$, we have

$$
\left|A(r, z)\left(t_{1}\right)-A(r, z)\left(t_{2}\right)\right|=0, \quad\left|B(r, z)\left(t_{1}\right)-B(r, z)\left(t_{2}\right)\right|=0 .
$$

Hence, $T(S)$ is equicontinuous. According to the Arzela-Ascoli theorem, $T(S)$ is a relative compact set.

So the operator $T$ is completely continuous on $E_{0}$.

Lemma 2.9 (see [2]) Let $E$ be a Banach space, and $P \subset E$ be a normal solid cone. Suppose that there exist $y_{1}, z_{1}, y_{2}, z_{2} \in E$, with $y_{1} \prec z_{1} \prec y_{2} \prec z_{2}$, and $A:\left[y_{1}, z_{2}\right] \rightarrow E$ is a completely continuous strongly increasing operator such that

$$
y_{1} \preceq A y_{1}, \quad A z_{1} \prec z_{1}, \quad y_{2} \preceq A y_{2}, \quad A z_{2} \preceq z_{2} .
$$

Then the operator $A$ has at least three fixed points $x_{1}, x_{2}, x_{3}$ such that

$$
y_{1} \preceq x_{1} \prec \prec z_{1}, \quad y_{2} \prec \prec x_{2} \preceq z_{2}, \quad y_{2} \npreceq x_{3} \npreceq z_{1} .
$$

\section{Multiple solutions of the coupled systems}

Definition 3.1 $(u, v) \in E_{0} \cap\left(A C^{2}(0,1) \times A C^{2}(0,1)\right)$ is called an upper solution of coupled system (2.13), if it satisfies

$$
\left\{\begin{array}{l}
D_{0^{+}}^{\alpha} u(t)+f\left(t, v(t), \psi_{t}+v_{t}\right) \leq 0, \quad t \in(0,1), \\
D_{0^{+}}^{\beta} \nu(t)+g\left(t, u(t), \phi_{t}+u_{t}\right) \leq 0, \quad t \in(0,1), \\
D_{0^{+}}^{\gamma_{1}} u(1) \leq a D_{0^{+}}^{\gamma_{1}} u(\xi), \quad D_{0^{+}}^{\gamma_{2}} v(1) \leq b D_{0^{+}}^{\gamma_{2}} v(\eta), \\
u(t)=0, \quad v(t)=0, \quad t \in[-\tau, 0] .
\end{array}\right.
$$

Definition 3.2 $(x, y) \in E_{0} \cap\left(A C^{2}(0,1) \times A C^{2}(0,1)\right)$ is called a lower solution of coupled system (2.13), if it satisfies

$$
\left\{\begin{array}{l}
D_{0^{+}}^{\alpha} x(t)+f\left(t, y(t), \psi_{t}+y_{t}\right) \geq 0, \quad t \in(0,1), \\
D_{0^{+}}^{\beta} y(t)+g\left(t, x(t), \phi_{t}+x_{t}\right) \geq 0, \quad t \in(0,1), \\
D_{0^{+}}^{\gamma_{1}} x(1) \geq a D_{0^{+}}^{\gamma_{1}} x(\xi), \quad D_{0^{+}}^{\gamma_{2}} y(1) \geq b D_{0^{+}}^{\gamma_{2}} y(\eta), \\
x(t)=0, \quad y(t)=0, \quad t \in[-\tau, 0] .
\end{array}\right.
$$

Lemma 3.1 Let $(u, v) \in E_{0} \cap\left(A C^{2}(0,1) \times A C^{2}(0,1)\right)$, if

$$
\left\{\begin{array}{l}
D_{0^{+}}^{\alpha} u(t) \leq 0, \quad t \in(0,1), \\
D_{0^{+}}^{\beta} \nu(t) \leq 0, \quad t \in(0,1), \\
D_{0^{+}}^{\gamma_{1}} u(1) \leq a D_{0^{+}}^{\gamma_{1}} u(\xi), \quad D_{0^{+}}^{\gamma_{2}} \nu(1) \leq b D_{0^{+}}^{\gamma_{2}} z(\eta), \\
u(t)=0, \quad v(t)=0, \quad t \in[-\tau, 0],
\end{array}\right.
$$

then $u(t) \geq 0$ and $v(t) \geq 0, t \in[0,1]$. 
Proof Let $D_{0^{+}}^{\alpha} u(t)=-x(t) \leq 0, D_{0^{+}}^{\alpha} v(t)=-y(t) \leq 0, D_{0^{+}}^{\gamma_{1}} u(1)-a D_{0^{+}}^{\gamma_{1}} u(\xi)=a_{0} \leq 0, D_{0^{+}}^{\gamma_{2}} z(1)-$ $b D_{0^{+}}^{\gamma_{2}} z(\eta)=b_{0} \leq 0$. Then $x(t) \geq 0, y(t) \geq 0, a_{0} \leq 0, b_{0} \leq 0$. By Lemma 2.3, the coupled system

$$
\left\{\begin{array}{l}
D_{0^{+}}^{\alpha} u(t)+x(t)=0, \quad t \in(0,1), \\
D_{0^{+}}^{\beta} v(t)+y(t)=0, \quad t \in(0,1), \\
u(0)=v(0)=0, \quad t \in[-\tau, 0], \\
D_{0^{+}}^{\gamma_{1}} u(1)=a D_{0^{+}}^{\gamma_{1}} u(\xi)-a_{0}, \quad D_{0^{+}}^{\gamma_{2}} \nu(1)=b D_{0^{+}}^{\gamma_{2}} \nu(\eta)-b_{0},
\end{array}\right.
$$

has a unique solution

$$
\begin{aligned}
& u(t)= \begin{cases}\int_{0}^{1} G_{\alpha}(t, s) x(s) d s-\frac{1}{\rho_{\alpha}} a_{0} \Gamma\left(\alpha-\gamma_{1}\right) t^{\alpha-1}, & t \in(0,1], \\
0, & t \in[-\tau, 0],\end{cases} \\
& v(t)= \begin{cases}\int_{0}^{1} G_{\beta}(t, s) y(s) d s-\frac{1}{\rho_{\beta}} b_{0} \Gamma\left(\beta-\gamma_{2}\right) t^{\beta-1}, & t \in(0,1], \\
0, & t \in[-\tau, 0] .\end{cases}
\end{aligned}
$$

By Lemma 2.4, $u(t) \geq 0$ and $v(t) \geq 0, t \in[0,1]$.

For convenience, we assume that the functions $f$ and $g$ satisfy the following properties.

$(\mathrm{H})$ For any $x_{1}, x_{2} \in[0,+\infty)$ with $0 \leq x_{1} \leq x_{2}$ and any $p_{1}, p_{2} \in C([-\tau, 0])$ with $p_{1} \leq p_{2}$,

$$
f\left(t, x_{1}, p_{1}\right) \leq f\left(t, x_{2}, p_{2}\right), \quad g\left(t, x_{1}, p_{1}\right) \leq g\left(t, x_{2}, p_{2}\right), \quad t \in[0,1]
$$

when at least $x_{1}<x_{2}$ and/or $p_{1}<p_{2}$ holds,

$$
f\left(t, x_{1}, p_{1}\right)<f\left(t, x_{2}, p_{2}\right), \quad g\left(t, x_{1}, p_{1}\right)<g\left(t, x_{2}, p_{2}\right), \quad t \in[0,1] .
$$

Lemma 3.2 Suppose $(H)$ holds, then $T$ is a strongly increasing operator.

Proof For any $\left(h_{1}, k_{1}\right),\left(h_{2}, k_{2}\right) \in E_{0}$ with $\left(h_{1}, k_{1}\right) \prec\left(h_{2}, k_{2}\right)$, i.e., $h_{1}(t) \leq h_{2}(t), k_{1}(t) \leq k_{2}(t)$ and $h_{1}(t) \not \equiv h_{2}(t)$ or $k_{1}(t) \not \equiv k_{2}(t)$ for $t \in[-\tau, 1]$.

By $(\mathrm{H})$, we have

$$
\begin{aligned}
& f\left(t, k_{1}(t), \psi_{t}+k_{1 t}\right) \leq f\left(t, k_{2}(t), \psi_{t}+k_{2 t}\right), \\
& g\left(t, h_{1}(t), \phi_{t}+h_{1 t}\right) \leq g\left(t, h_{2}(t), \phi_{t}+h_{2 t}\right), \quad t \in[0,1] .
\end{aligned}
$$

Since $h_{1}(t) \not \equiv h_{2}(t)$ and $k_{1}(t) \not \equiv k_{2}(t)$, there exist two intervals $\left[a_{1}, b_{1}\right],\left[a_{2}, b_{2}\right] \subset[0,1]$ such that $k_{1}(t)<k_{2}(t)$ for $t \in\left[a_{1}, b_{1}\right]$ or $h_{1}(t)<h_{2}(t)$ for $t \in\left[a_{2}, b_{2}\right]$. Then

$$
f\left(t, k_{1}(t), \psi_{t}+k_{1 t}\right)<f\left(t, k_{2}(t), \psi_{t}+k_{2 t}\right), \quad t \in\left[a_{1}, b_{1}\right],
$$

and

$$
g\left(t, h_{1}(t), \phi_{t}+h_{1 t}\right)<g\left(t, h_{2}(t), \phi_{t}+h_{2 t}\right), \quad t \in\left[a_{2}, b_{2}\right] .
$$


From (2.14), (2.15), (3.3), (3.4) and Lemma 2.4, for any $t \in(0,1]$,

$$
\begin{aligned}
A & \left(h_{2}, k_{2}\right)(t)-A\left(h_{1}, k_{1}\right)(t) \\
\quad & =\int_{0}^{1} G_{\alpha}(t, s) f\left(s, k_{2}(s), \psi_{s}+k_{2 s}\right) d s-\int_{0}^{1} G_{\alpha}(t, s) f\left(s, k_{1}(s), \psi_{s}+k_{1 s}\right) d s>0, \\
B & \left(h_{2}, k_{2}\right)(t)-B\left(h_{1}, k_{1}\right)(t) \\
\quad & \int_{0}^{1} G_{\beta}(t, s) g\left(s, h_{2}(s), \phi_{s}+h_{2 s}\right) d s-\int_{0}^{1} G_{\beta}(t, s) g\left(s, h_{1}(s), \phi_{s}+h_{1 s}\right) d s>0 .
\end{aligned}
$$

For any $t \in[-\tau, 0]$, we have

$$
\begin{aligned}
& A\left(h_{2}, k_{2}\right)(t)-A\left(h_{1}, k_{1}\right)(t)=0, \\
& B\left(h_{2}, k_{2}\right)(t)-B\left(h_{1}, k_{1}\right)(t)=0 .
\end{aligned}
$$

In conclusion, we have $T\left(h_{1}, k_{1}\right) \prec \prec T\left(h_{2}, k_{2}\right), T$ is a strongly increasing operator.

Theorem 3.3 Suppose $(H)$ holds, and there exist two lower solutions $\left(x_{1}, y_{1}\right),\left(x_{2}, y_{2}\right)$ and two upper solutions $\left(u_{1}, v_{1}\right),\left(u_{2}, v_{2}\right)$ of coupled system $(2.13)$ such that $\left(x_{1}, y_{1}\right),\left(u_{2}, v_{2}\right)$ are not solutions of the coupled system (2.13) with

$$
\left(x_{1}, y_{1}\right) \prec\left(u_{1}, v_{1}\right) \prec\left(x_{2}, y_{2}\right) \prec\left(u_{2}, v_{2}\right) .
$$

Then the coupled system (1.1) has at least three distinct solutions $\left(r_{1}+\phi, z_{1}+\psi\right),\left(r_{2}+\phi, z_{2}+\right.$ $\psi),\left(r_{3}+\phi, z_{3}+\psi\right) \in E$ and for $t \in[0,1]$,

$$
\begin{aligned}
& \left(x_{1}(t), y_{1}(t)\right) \leq\left(r_{1}(t), z_{1}(t)\right)<\left(u_{1}(t), v_{1}(t)\right), \\
& \left(x_{2}(t), y_{2}(t)\right)<\left(r_{2}(t), z_{2}(t)\right) \leq\left(u_{2}(t), v_{2}(t)\right), \\
& \left(u_{2}(t), v_{2}(t)\right) 太\left(r_{3}, z_{3}\right) 太\left(u_{1}(t), v_{1}(t)\right) .
\end{aligned}
$$

Proof By Lemma 2.8 and Lemma 3.2, we see that $T: E_{0} \rightarrow E_{0}$ is a completely continuous strongly increasing operator.

Let $A\left(x_{1}, y_{1}\right):=x_{1}^{(1)}, B\left(x_{1}, y_{1}\right):=y_{1}^{(1)}$, then from the definition of $T$,

$$
\left\{\begin{array}{l}
D_{0^{+}}^{\alpha} x_{1}^{(1)}(t)+f\left(t, y_{1}(t), \psi_{t}+y_{1 t}\right)=0, \quad t \in(0,1), \\
D_{0^{+}}^{\beta} y_{1}^{(1)}(t)+g\left(t, x_{1}(t), \phi_{t}+x_{1 t}\right)=0, \quad t \in(0,1), \\
D_{0^{+}}^{\gamma_{1}} x_{1}^{(1)}(1)=a D_{0^{+}}^{\gamma_{1}} x_{1}(\xi), \quad D_{0^{+}}^{\gamma_{2}} y_{1}^{(1)}(1)=b D_{0^{+}}^{\gamma_{2}} y_{1}(\eta), \\
x_{1}^{(1)}(t)=0, \quad y_{1}^{(1)}(t)=0, \quad t \in[-\tau, 0] .
\end{array}\right.
$$

By (3.2) and (3.8),

$$
\begin{aligned}
D_{0^{+}}^{\alpha}\left(x_{1}(t)-x_{1}^{(1)}(t)\right) & =D_{0^{+}}^{\alpha} x_{1}(t)-D_{0^{+}}^{\alpha} x_{1}^{(1)}(t) \\
& \geq-f\left(t, y_{1}(t), \psi_{t}+y_{1 t}\right)+f\left(t, y_{1}(t), \psi_{t}+y_{1 t}\right)=0
\end{aligned}
$$




$$
\begin{aligned}
D_{0^{+}}^{\alpha}\left(y_{1}(t)-y_{1}^{(1)}(t)\right) & =D_{0^{+}}^{\alpha} y_{1}(t)-D_{0^{+}}^{\alpha} y_{1}^{(1)}(t) \\
& \geq-g\left(t, x_{1}(t), \phi_{t}+x_{1 t}\right)+g\left(t, x_{1}(t), \phi_{t}+x_{1 t}\right)=0 . \\
D_{0^{+}}^{\gamma_{1}}\left(x_{1}(1)-x_{1}^{(1)}(1)\right) & \geq a D_{0^{+}}^{\gamma_{1}} x_{1}(\xi)-a D_{0^{+}}^{\gamma_{1}} x_{1}(\xi), \\
D_{0^{+}}^{\gamma_{2}}\left(y_{1}(1)-y_{1}^{(1)}(1)\right) & \geq b D_{0^{+}}^{\gamma_{2}} y_{1}(\eta)-b D_{0^{+}}^{\gamma_{2}} y_{1}(\eta) .
\end{aligned}
$$

It is clear that

$$
x_{1}^{(1)}(t)-x_{1}(t)=0, \quad y_{1}^{(1)}(t)-y_{1}(t)=0, \quad t \in[-\tau, 0] .
$$

By Lemma 3.1,

$$
x_{1}^{(1)}(t)-x_{1}(t) \geq 0, \quad y_{1}^{(1)}(t)-y_{1}(t) \geq 0, \quad t \in[0,1] .
$$

Therefore,

$$
\left(x_{1}, y_{1}\right) \preceq T\left(x_{1}, y_{1}\right) .
$$

Similarly, we can prove $T\left(x_{2}, y_{2}\right) \preceq\left(x_{2}, y_{2}\right)$. Because $\left(x_{2}, y_{2}\right)$ is a lower solution of coupled system (2.13) and not a solution of (2.13), we have $T\left(x_{2}, y_{2}\right) \neq\left(x_{2}, y_{2}\right)$. Thus

$$
T\left(x_{2}, y_{2}\right) \prec\left(x_{2}, y_{2}\right) .
$$

In the same way, we get

$$
T\left(u_{1}, v_{1}\right) \prec\left(u_{1}, v_{1}\right), \quad T\left(u_{2}, v_{2}\right) \preceq\left(u_{2}, v_{2}\right) .
$$

It follows that $T$ has at least three fixed points $\left(r_{1}, z_{1}\right),\left(r_{2}, z_{2}\right),\left(r_{3}, z_{3}\right) \in\left[\left(x_{1}, y_{1}\right),\left(u_{2}, v_{2}\right)\right]$ from Lemma 2.9.

Hence, by Lemma 2.6, the coupled system (1.1) has at least three distinct solutions $\left(r_{1}+\right.$ $\left.\phi, z_{1}+\psi\right),\left(r_{2}+\phi, z_{2}+\psi\right),\left(r_{3}+\phi, z_{3}+\psi\right) \in E$, and for $t \in[0,1]$

$$
\begin{aligned}
& \left(x_{1}(t), y_{1}(t)\right) \leq\left(r_{1}(t), z_{1}(t)\right)<\left(u_{1}(t), v_{1}(t)\right), \\
& \left(x_{2}(t), y_{2}(t)\right)<\left(r_{2}(t), z_{2}(t)\right) \leq\left(u_{2}(t), v_{2}(t)\right), \\
& \left(u_{2}(t), v_{2}(t)\right) 太\left(r_{3}, z_{3}\right) 太\left(u_{1}(t), v_{1}(t)\right) .
\end{aligned}
$$

\section{Illustration}

To illustrate the applicability of the conclusion, we consider the following nonlinear differential fractional coupled system:

$$
\begin{cases}D_{0^{+}}^{\frac{3}{2}} u(t)+\frac{1}{\pi} \arctan (\sqrt{t} v(t))+0.01\left\|v_{t}\right\|_{\tau}=0, & t \in[0,1], \\ D_{0^{+}}^{\frac{5}{4}} \nu(t)+\frac{1}{\pi} \arctan \left(t^{\frac{1}{4}} u(t)\right)+0.01\left\|u_{t}\right\|_{\tau}=0, \quad t \in[0,1], & \\ u(t)=t^{2}, \quad v(t)=t^{4}, \quad t \in\left[-\frac{1}{2}, 0\right], \\ D_{0^{+}}^{\frac{3}{8}} u(1)=D_{0^{+}}^{\frac{3}{8}} u\left(\frac{1}{2}\right), \quad D_{0^{+}}^{\frac{5}{8}} \nu(1)=\frac{29}{50} D_{0^{+}}^{\frac{5}{8}} \nu\left(\frac{1}{4}\right) .\end{cases}
$$


The coupled system (4.1) can be regarded as the form of (1.1), where $\alpha=\frac{3}{2}, \beta=\frac{5}{4}$, $\tau=\frac{1}{2}, \gamma_{1}=\frac{3}{8}, \gamma_{2}=\frac{5}{8}, \xi=\frac{1}{2}, \eta=\frac{1}{4}, a=1, b=\frac{3}{5}, f\left(t, v(t), v_{t}\right)=\frac{1}{\pi} \arctan (\sqrt{t} v(t))+0.01\left\|v_{t}\right\|_{\tau}$, $g\left(t, u(t), u_{t}\right)=\frac{1}{\pi} \arctan \left(t^{\frac{1}{4}} u(t)\right)+0.01\left\|u_{t}\right\|_{\tau}, \varphi(t)=t^{2}, \psi(t)=t^{4}$.

It is obvious that

$$
\begin{aligned}
1-a \xi^{\alpha-\gamma_{1}-1} & =1-\left(\frac{1}{2}\right)^{\frac{3}{2}-\frac{3}{8}-1} \approx 0.082996>0,1-b \eta^{\beta-\gamma_{2}-1} \\
& =1-\frac{29}{50}\left(\frac{1}{4}\right)^{\frac{5}{4}-\frac{5}{8}-1} \approx 0.02456>0
\end{aligned}
$$

Take

$$
\begin{aligned}
& u_{1}(t)=\left\{\begin{array}{ll}
\frac{1}{\sqrt{\pi}}(16-2 t) \sqrt{t}, & t \in[0,1], \\
t^{2}, & t \in\left[-\frac{1}{2}, 0\right],
\end{array} \quad v_{1}(t)= \begin{cases}\frac{1}{\Gamma(0.25)}(10-2 t) t^{\frac{1}{4},} & t \in[0,1], \\
t^{4}, & t \in\left[-\frac{1}{2}, 0\right],\end{cases} \right. \\
& u_{2}(t)=\left\{\begin{array}{ll}
\frac{1}{\sqrt{\pi}}(60-8 t) \sqrt{t}, & t \in[0,1], \\
t^{2}, & t \in\left[-\frac{1}{2}, 0\right],
\end{array} \quad v_{2}(t)= \begin{cases}\frac{1}{\Gamma(0.25)}(80-3 t) t^{\frac{1}{4},} & t \in[0,1], \\
t^{4}, & t \in\left[-\frac{1}{2}, 0\right],\end{cases} \right. \\
& x_{1}(t)=\left\{\begin{array}{ll}
0, & t \in[0,1], \\
t^{2}, & t \in\left[-\frac{1}{2}, 0\right],
\end{array} \quad y_{1}(t)= \begin{cases}0, & t \in[0,1], \\
t^{4}, & t \in\left[-\frac{1}{2}, 0\right]\end{cases} \right. \\
& x_{2}(t)=\left\{\begin{array}{ll}
\frac{15 \sqrt{\pi}}{\Gamma\left(\frac{15}{8}\right)}\left(5-4 t^{\frac{1}{16}}\right) t^{\frac{7}{8}}, & t \in[0,1], \\
t^{2}, & t \in\left[-\frac{1}{2}, 0\right],
\end{array} y_{2}(t)= \begin{cases}\frac{t^{\frac{3}{8}}}{\Gamma\left(\frac{5}{8}\right)}\left(23-\frac{8}{5} t^{\frac{3}{4}}\right), & t \in[0,1], \\
t^{4}, & t \in\left[-\frac{1}{2}, 0\right] .\end{cases} \right.
\end{aligned}
$$

By simple computations, we have

$$
\begin{aligned}
& \left\{\begin{array}{l}
D_{0^{+}}^{\frac{3}{2}} u_{1}(t)+f\left(t, v_{1}(t), v_{1 t}+\psi_{t}\right)=-\frac{3}{2}+f\left(t, v_{1}(t), v_{1 t}+\psi_{t}\right) \leq 0, \quad t \in[0,1], \\
D_{0^{+}}^{\frac{5}{4}} v_{1}(t)+g\left(t, u_{1}(t), u_{1 t}+\phi_{t}\right) \approx-0.625+g\left(t, u_{1}(t), u_{1 t}+\phi_{t}\right) \leq 0, \quad t \in[0,1], \\
u_{1}(t)=0, \quad v_{1}(t)=0, \quad t \in\left[-\frac{1}{2}, 0\right], \\
D_{0^{+}}^{\frac{3}{8}} u_{1}(1) \approx 7.07907 \leq D_{0^{+}}^{\frac{3}{8}} u_{1}\left(\frac{1}{2}\right) \approx 7.14069, \\
D_{0^{+}}^{\frac{5}{8}} v_{1}(1) \approx 1.04565 \leq \frac{29}{50} D_{0^{+}}^{\frac{5}{8}} \nu_{1}\left(\frac{1}{4}\right) \approx 1.52995,
\end{array}\right. \\
& \left\{\begin{array}{l}
D_{0^{+}}^{\frac{3}{2}} u_{2}(t)+f\left(t, v_{2}(t), v_{2 t}+\psi_{t}\right)=-6+f\left(t, v_{2}(t), v_{2 t}+\psi_{t}\right) \leq 0, \quad t \in[0,1], \\
D_{0^{+}}^{\frac{5}{4}} v_{2}(t)+g\left(t, u_{2}(t), u_{2 t}+\phi_{t}\right) \approx-0.9375+g\left(t, u_{2}(t), u_{2 t}+\phi_{t}\right) \leq 0, \quad t \in[0,1], \\
u_{2}(t)=0, v_{2}(t)=0, \quad t \in\left[-\frac{1}{2}, 0\right], \\
D_{0^{+}}^{\frac{3}{8}} u_{2}(1) \approx 26.19258 \leq D_{0^{+}}^{\frac{3}{8}} u_{2}\left(\frac{1}{2}\right) \approx 26.61531, \\
D_{0^{+}}^{\frac{5}{8}} v_{2}(1) \approx 12.89631 \leq \frac{29}{50} D_{0^{+}}^{\frac{5}{8}} \nu_{2}\left(\frac{1}{4}\right) \approx 13.34455,
\end{array}\right. \\
& \left\{\begin{array}{l}
\frac{3}{D_{0^{+}}^{2}} x_{1}(t)+f\left(t, y_{1}(t), y_{1 t}+\psi_{t}\right)=0+f\left(t, y_{1}(t), y_{1 t}+\psi_{t}\right) \geq 0, \quad t \in[0,1], \\
D_{0^{+}}^{\frac{5}{4}} y_{1}(t)+g\left(t, x_{1}(t), x_{1 t}+\phi_{t}\right)=0+g\left(t, x_{1}(t), x_{1 t}+\phi_{t}\right) \geq 0, \quad t \in[0,1], \\
x_{1}(t)=0, \quad y_{1}(t)=0, \quad t \in\left[-\frac{1}{2}, 0\right], \\
D_{0^{+}}^{\frac{3}{8}} x_{1}(1)=0 \geq D_{0^{+}}^{\frac{3}{8}} x_{1}\left(\frac{1}{2}\right)=0, \\
D_{0^{+}}^{\frac{5}{8}} y_{1}(1)=0 \geq \frac{29}{50} D_{0^{+}}^{\frac{5}{8}} y_{1}\left(\frac{1}{4}\right)=0,
\end{array}\right.
\end{aligned}
$$




$$
\left\{\begin{array}{l}
D_{0^{+}}^{\frac{3}{2}} x_{2}(t)+f\left(t, y_{2}(t), y_{2 t}+\psi_{t}\right) \\
\quad=\frac{5 \pi}{64 \Gamma\left(\frac{1}{2}\right)}\left(99 t^{\frac{-5}{8}}\left(\frac{5}{\Gamma\left(\frac{19}{8}\right)}-\frac{4 t \frac{1}{16} \Gamma\left(\frac{31}{16}\right)}{\Gamma\left(\frac{15}{8}\right) \Gamma\left(\frac{39}{16}\right)}\right)-\frac{87 \Gamma\left(\frac{31}{16}\right) t \frac{-9}{16}}{\Gamma\left(\frac{15}{8}\right) \Gamma\left(\frac{39}{16}\right)}\right)+f\left(t, y_{2}(t), y_{2 t}+\psi_{t}\right) \\
\geq 0, \quad t \in[0,1], \\
D_{0^{+}}^{\frac{5}{4}} y_{2}(t)+g\left(t, x_{2}(t), x_{2 t}+\phi_{t}\right) \\
\quad=\left(\frac{1842^{\frac{1}{4}} \Gamma\left(\frac{11}{8}\right)}{\sqrt{\pi} \Gamma\left(\frac{5}{4}\right)}-\frac{9 t^{\frac{3}{4}} \Gamma\left(\frac{17}{8}\right)}{\Gamma\left(\frac{13}{8}\right) \Gamma\left(\frac{23}{8}\right)}\right) 64 t^{\frac{-7}{8}}-\frac{3 \Gamma\left(\frac{17}{8}\right) t^{\frac{-1}{8}}}{2 \Gamma\left(\frac{13}{8}\right) \Gamma\left(\frac{23}{8}\right)}+g\left(t, x_{2}(t), x_{2 t}+\phi_{t}\right) \\
\geq 0, \quad t \in[0,1], \\
x_{2}(t)=0, \quad y_{2}(t)=0, \quad t \in\left[-\frac{1}{2}, 0\right], \\
D_{0^{+}}^{\frac{3}{8}} x_{2}(1) \approx 27.76526 \geq D_{0^{+}}^{\frac{3}{8}} x_{2}\left(\frac{1}{2}\right) \approx 23.29748, \\
D_{0^{+}}^{\frac{5}{8}} y_{2}(1) \approx 10.29709 \geq \frac{29}{50} D_{0^{+}}^{\frac{5}{8}} y_{2}\left(\frac{1}{4}\right) \approx 9.15314,
\end{array}\right.
$$

which show $\left(u_{1}, v_{1}\right)$ and $\left(u_{2}, u_{2}\right)$ are upper solutions, $\left(x_{1}, y_{1}\right)$ and $\left(x_{2}, y_{2}\right)$ are lower solutions of the coupled systems (4.1), and it is not hard to get $\left(x_{1}, y_{1}\right) \prec\left(u_{1}, v_{1}\right) \prec\left(x_{2}, y_{2}\right) \prec\left(u_{2}, v_{2}\right)$.

It is easy to obtain

$$
\begin{aligned}
0 & \leq f\left(t, v_{2}(t), v_{2 t}+\psi_{t}\right)-f\left(t, v_{1}(t), v_{1 t}+\phi_{t}\right) \\
& \leq \frac{1}{\pi}\left\|v_{2}-v_{1}\right\|+0.01\left\|\left(v_{2 t}+\psi_{t}\right)-\left(v_{1 t}+\phi_{t}\right)\right\|_{\tau} \leq\left(\frac{1}{\pi}+0.01\right)\left\|v_{2}-v_{1}\right\|
\end{aligned}
$$

and

$$
\begin{aligned}
0 & \leq g\left(t, u_{2}(t), u_{2 t}+\psi_{t}\right)-g\left(t, u_{1}(t), u_{1 t}+\phi_{t}\right) \\
& \left.\leq \frac{1}{\pi}\left\|u_{2}-u_{1}\right\|+0.01 \| u_{2 t}+\psi_{t}\right)-\left(u_{2 t}+\phi_{t}\right)\left\|_{\tau} \leq\left(\frac{1}{\pi}+0.01\right)\right\| u_{2}-u_{1} \|
\end{aligned}
$$

Similarly,

$$
f\left(t, y_{2}(t), y_{2 t}+\psi_{t}\right) \geq f\left(t, y_{1}(t), y_{1 t}+\psi_{t}\right), \quad g\left(t, x_{2}(t), x_{2 t}+\phi_{t}\right) \geq g\left(t, x_{1}(t), x_{1 t}+\phi_{t}\right)
$$

Then condition $(\mathrm{H})$ is satisfied. And all conditions of Theorem 3.3 are satisfied. In view of Theorem 3.3, the coupled system (4.1) has at least three distinct solutions $\left(r_{1}+\phi, z_{1}+\right.$ $\psi),\left(r_{2}+\phi, z_{2}+\psi\right),\left(r_{3}+\phi, z_{3}+\psi\right) \in\left[\left(x_{1}, y_{1}\right),\left(u_{2}, v_{2}\right)\right]$ and, moreover,

$$
\begin{aligned}
& \left(x_{1}(t), y_{1}(t)\right) \leq\left(r_{1}(t), z_{1}(t)\right)<\left(u_{1}(t), v_{1}(t)\right), \\
& \left(x_{2}(t), y_{2}(t)\right)<\left(r_{2}(t), z_{2}(t)\right) \leq\left(u_{2}(t), v_{2}(t)\right), \\
& \left(u_{2}(t), v_{2}(t)\right) 太\left(r_{3}(t), z_{3}(t)\right) 太\left(u_{1}(t), v_{1}(t)\right) .
\end{aligned}
$$

\section{Conclusion}

In this paper, we present the method of upper and lower solutions for a class of fractional coupled systems including state dependent delays with nonlocal boundary conditions. By using the method of upper and lower solutions and fixed point theorems on the normal cone, the multiplicity results for the boundary value problem are established. The method and main results obtained in this paper can also be extended to the boundary value prob- 
lems of function fractional differential high dimensional systems of the form

$$
\left\{\begin{array}{l}
D_{0^{+}}^{\alpha_{1}} u_{1}(t)+f_{1}\left(t, u_{2}(t), u_{2 t}\right)=0, \quad t \in(0,1), \\
D_{0^{+}}^{\alpha_{2}} u_{2}(t)+f_{2}\left(t, u_{3}(t), u_{3 t}\right)=0, \quad t \in(0,1), \\
\cdots \\
D_{0^{+}}^{\alpha_{m}} u_{m}(t)+f_{m}\left(t, u_{1}(t), u_{1 t}\right)=0, \quad t \in(0,1), \\
u_{i}(t)=\varphi_{i}(t), \quad t \in[-\tau, 0], \quad i=1,2, \cdots, m, \\
D_{0^{+}}^{\gamma_{i}} u_{i}(1)=a_{i} D_{0^{+}}^{\gamma_{i}} u_{i}\left(\xi_{i}\right), \quad i=1,2, \cdots, m .
\end{array}\right.
$$

\section{Acknowledgements}

The authors sincerely thank the editors and anonymous referees for the careful reading of the original manuscript and valuable comments, which have improved the quality of our work.

Funding

This work is supported by the National Natural Science Foundation of China (No.11171220).

Availability of data and materials

Data sharing not applicable to this article as no datasets were generated or analysed during the current study.

\section{Competing interests}

The authors declare that they have no competing interests.

\section{Authors' contributions}

The authors declare that the work was realized in collaboration with the same responsibility. All authors read and approved the final manuscript.

\section{Publisher's Note}

Springer Nature remains neutral with regard to jurisdictional claims in published maps and institutional affiliations.

Received: 5 November 2020 Accepted: 9 May 2021 Published online: 19 May 2021

\section{References}

1. Kilbas, A.A., Srivastava, H.M., Trujillo, J.J.: Theory and Applications of Fractional Differential Equations, pp. 69-79. Elsevier, Amsterdam (2006)

2. Gou, D.J., Sun, J.X., Liu, Z.L.: Functional Methods for Nonlinear Ordinary Differential Equations. pp. 219-222. Shandong Science and Technology Press (In Chinese) (2005)

3. Podlubny, I.: Fractional Differential Equations. Academic Press, San Diego (1999)

4. Tian, Y.S., Bai, Z.B., Sun, S.J.: Positive solutions for a boundary value problem of fractional differential equation with p-Laplacian operator. Adv. Differ. Equ. 2019, 349 (2019)

5. Qiao, Y., Chen, F.Q., An, Y.K.: Existence and multiplicity of weak solutions for a class of fractional Sturm-Liouville boundary value problems with impulsive conditions. J. Appl. Anal. 10, 2537-2556 (2020)

6. Matar, M.M., Abu, S.E., Alzabut, J.: On solvability of nonlinear fractional differential systems involving nonlocal initial conditions. Math. Methods Appl. Sci. 2019, 1-12 (2019)

7. Du, X.R., Meng, Y., Pang, H.H.: Iterative positive solutions to a coupled Hadamard-type fractional differential system on infinite domain with the multistrip and multipoint mixed boundary conditions. J. Funct. Spaces 2020, Article ID 6508075 (2020)

8. Liu, X.P., Jia, M.: Solvability and numerical simulations for BVPs of fractional coupled systems involving left and right fractional derivatives. Appl. Math. Comput. 353, 230-242 (2019)

9. Ali, Z., Zada, A., Shah, K.: On Ulam stability for a coupled systems of nonlinear implicit fractional differential differential equations. Bull. Malays. Math. Sci. Soc. 42, 2681-2699 (2019)

10. Zhang, Y., Bai, Z., Feng, T.: Existence results for a coupled system of nonlinear fractional three-point boundary value problems at resonance. Comput. Math. Appl. 61, 1032-1047 (2011)

11. Asif, N.A., Khao, R.A.: Positive solutions to singular system with four-point coupled boundary conditions. J. Math. Anal. Appl. 386, 848-861 (2012)

12. Hou, Y.M., Zhang, L.H., Wang, G.T.: A new comparison principle and its application to nonlinear impulsive functional integro-differential equations. Adv. Differ. Equ. 2008, 380 (2008)

13. Chegis, R.: Numerical solution of a heat conduction problem with an integral conduction. Liet. Mat. Rink. 24, 209-215 (1984)

14. Faieghi, M., Kuntanapreeda, S., Delavari, H., et al.: LMI-based stabilization of a class of fractional-order chaotic systems. Nonlinear Dyn. Syst. Theory 72, 301-309 (2013)

15. Ionkin, N.I.: Solution of a boundary value problem in heat conduction with a nonclassical boundary condition. Differ. Equ. 13, 294-304 (1977)

16. Ahmad, B., Ntouyas, S.K., Alsaedi, A.: Existence results for a system of coupled hybrid fractional differential equations. Abstr. Appl. Anal. 2014, Article ID 672167 (2014) 
17. Zhang, F.: The synchronization of a fractional-order chaotic system. Adv. Math. Res. 655, 1488-1491 (2013)

18. Zhang, X., Liu, L., Wu, Y.: The uniqueness of positive solution for a singular fractional differential system involving derivatives. Commun. Nonlinear Sci. Numer. Simul. 18, 1400-1409 (2013)

19. Ahmad, M., Zada, A., Alzabut, J.: Hyers-Ulam stability of a coupled system of fractional differential equations of Hilfer-Hadamard type. Demonstr. Math. 52, 283-295 (2019)

20. Singh, J., Rashidi, M.M., Kumar, D., et al.: A fractional model of a dynamical Brusselator reaction-diffusion system arising in triple collision and enzymatic reactions. Nonlinear Eng. 5, 277-285 (2016)

21. Bourguiba, R., Rodriguez-Lopez, R.: Existence results for fractional differential equations in presence of upper and lower solutions. Discrete Contin. Dyn. Syst., Ser. B 26, 1723-1747 (2021)

22. Chen, H.L., Meng, S.M., Cui, Y.J.: Monotone iterative technique for conformable fractional differential equations with deviating arguments. Discrete Dyn. Nat. Soc. 2020, 1-9 (2020)

23. Liu, X.P., Jia, M.: The method of lower and upper solutions for the general boundary value problems of fractional differential equations with p-Laplacian. Adv. Differ. Equ. 2018, 28 (2018)

24. Jia, M., Liu, X.P.: Multiplicity of solutions for integral boundary value problems of fractional differential equations with upper and lower solutions. Appl. Math. Comput. 232, 313-323 (2014)

25. Liu, X.P., Jia, M., Ge, W.G.: The method of lower and upper solutions for mixed fractional four-point boundary value problem with p-Laplacian operator. Appl. Math. Lett. 65, 56-62 (2017)

26. Zheng, Z.X.: Theory of Functional Differential Equations Education Press, Anhui (1994) (In Chinese)

27. Zheng, Z.X.: Development and application of functional differential equations. Adv. Math. 12, 94-112 (1983) (In (hinese)

28. Peng, S.G., Zhu, S.M.: Positive solutions of periodic boundary value problem for functional differential equations. Chin Ann. Math. Ser. B 26, 419-426 (2005)

29. Su, X.W.: Positive solutions to singular boundary value problems for fractional functional differential equations with changing sign nonlinearity. Comput. Math. Appl. 64, 3425-3435 (2012)

30. Zhao, K.H., Wang, K.: Existence of solutions for the delayed nonlinear fractional functional differential equations with three-point integral boundary value conditions. Adv. Differ. Equ. 2016, 284 (2016)

31. Zhu, B., Liu, L.S., Wu, Y.H.: Existence and uniqueness of global mild solutions for a class of nonlinear fractional reaction-diffusion equations with delay. Comput. Math. Appl. 78, 1811-1818 (2019)

\section{Submit your manuscript to a SpringerOpen ${ }^{\circ}$ journal and benefit from:}

- Convenient online submission

- Rigorous peer review

- Open access: articles freely available online

- High visibility within the field

Retaining the copyright to your article

Submit your next manuscript at $\gg$ springeropen.com 